Geistige Eigentumsrechte, was steht für die Entwicklungsländer auf dem spiel?

\title{
Patente im pharmazeutischen Sektor : Standpunkt der schweizerischen Industrie
}

Brian A. Yorke and Philip W. Grubb

\section{(2) OpenEdition Journals}

Electronic version

URL: http://journals.openedition.org/sjep/691

DOI: 10.4000/sjep.691

ISSN: 1663-9677

\section{Publisher}

Institut de hautes études internationales et du développement

\section{Printed version}

Date of publication: 1 avril 1998

Number of pages: 107-117

ISSN: 1660-5926

\section{Electronic reference}

Brian A. Yorke und Philip W. Grubb, «Patente im pharmazeutischen Sektor : Standpunkt der schweizerischen Industrie », Schweizerisches Jahrbuch für Entwicklungspolitik [Online], 17 | 1998, Online erschienen am: 03 August 2012, abgerufen am 08 September 2020. URL : http:// journals.openedition.org/sjep/691; DOI : https://doi.org/10.4000/sjep.691 


\title{
PATENTE IM PHARMAZEUTISCHEN SEKTOR: STANDPUNKT DER SCHWEIZERISCHEN INDUSTRIE
}

\author{
B. A. YORKE ${ }^{*}$ UND P. W. GRUB ${ }^{* *}$
}

N DEN letzten Jahren wurde der Patentschutz für Arzneimittel insbesondere in den Entwicklungsländern erheblich gestärkt. Die pharmazeutische Industrie begrüsst diese Verbesserung, die weitgehend auf die Verabschiedung des

TRIPs-Abkommens im Rahmen des GATT zurückgeht. Der vorliegende Artikel befasst sich mit den im TRIPs verlangten patentrechtlichen Änderungen, wobei der Schwerpunkt auf den Entwicklungsländern liegt, und gibt einen Überblick über die Umsetzung des TRIPs-Abkommens.

Das Allgemeine Zoll- und Handelsabkommen (GATT) wurde im Jahr 1948 zur Regelung der multilateralen Handelsfragen unterzeichnet. Die letzte GATT-Verhandlungsrunde, die Uruguay-Runde, wurde im April 1994 abgeschlossen und führte zur Errichtung der Welthandelsorganisation (WTO), die ihre Arbeiten im Januar 1995 aufnahm. Das Abkommen über handelsbezogene Aspekte der Rechte an geistigem Eigentum (TRIPs) wurde als wesentlicher Bestandteil der Schlussakte der Uruguay-Runde angenommen: Alle Länder, welche die neuen GATT-Verträge ratifizieren und WTO-Mitglieder werden, müssen demnach die TRIPs-Bestimmungen als Teil des Pakets übernehmen.

Das TRIPs-Abkommen deckt eine breite Palette von Fragen zum geistigen Eigentum ab, darunter Patente, Marken, geographische Angaben, gewerbliche Muster, integrierte Schaltkreise, Urheberrechte, Schutz vertraulicher Geschäftsinformationen sowie allgemeine Bestimmungen zu Grundsätzen, Durchführung und Streitbeilegung. Der folgende Kommentar beschränkt sich auf Patente, insbesondere in bezug auf Arzneimittel.

\section{BEDEUTUNG VON GATT/TRIPS}

\subsection{GRUNDLEGENDE PRINZIPIEN DES TRIPS-ABKOMMENS}

$\mathrm{Zu}$ den Grundsätzen des TRIPs-Abkommens gehören die Inländerbehandlung und die Meistbegünstigung. Demnach darf kein Mitglied die Staatsangehörigen der anderen Mitglieder weniger günstig behandeln als seine eigenen; jedes Mitglied muss den Staatsangehörigen aller Mitglieder sämtliche Privilegien gewähren, die es den Staatsangehörigen eines bestimmten Mitglieds gewährt. So sollen abgesehen von bestimmten Ausnahmen bilaterale Abkommen zwischen Mitgliedstaaten künftig nicht mehr gestattet sein. Diese Artikel sind besonders wichtig, denn im Unterschied zu anderen TRIPs-Bestimmungen darf kein Mitgliedsstaat (unabhängig von seinem Entwicklungsstand) die Anwendung über den 1. Januar 1996 hinaus aufschieben.

* Director, Corporate Intellectual Property, Novartis International AG, Basel.

** Intellectual Property Consel, Novartis Internatioal AG, CH-4002 Basel. 


\subsection{SPEZIFISCHE BESTIMMUNGEN ZU PATENTEN}

Das TRIPs-Abkommen fordert die WTO-Mitgliedsstaaten auf, einen wirksamen Patentschutz einzuführen. Hauptelemente des Abkommens sind:

口 Die Patentvergabe soll in allen Technologiebereichen, einschliesslich Stoffschutzpatente für Arzneimittel, im wesentlichen den im Europäischen Patentübereinkommen niedergelegten Patentierungskriterien entsprechen. Die Mitglieder können bestimmte Kategorien von Erfindungen, z.B. medizinische Behandlungsmethoden und - zumindest vorläufig noch - Pflanzen und Tiere, von der Patentfähigkeit ausschliessen. Allerdings fehlt z.B. eine Bestimmung, wonach die Patentfähigkeit eines menschlichen Gens ausgenommen werden könnte, sofern es die normalen Kriterien der Neuheit, erfinderischen Tätigkeit und gewerblichen Anwendbarkeit erfüllt. Dabei ist zu bedenken, dass die Schweiz mit der Annahme der Genschutzinitiative gegen ihre Verpflichtungen aus dem GATT/TRIPs verstossen würde.

\ Bei der Erteilung von Patenten und der Ausübung von Patentrechten müssen eingeführte und vor Ort produzierte Erzeugnisse gleich behandelt werden. Demnach ist es künftig nicht mehr möglich, Zwangslizenzen zu erteilen oder Patente für ein nicht lokal erzeugtes Produkt zu widerrufen, sofern der inländische Markt ausreichend mit Importen dieses im Ausland hergestellten Produktes versorgt ist.

- Der Import eines patentrechtlich geschützten Produkts sowie die Verwendung, der Verkauf oder der Import eines unmittelbar auf ein patentiertes Verfahren zurückgehenden Produkts gelten als Verstoss gegen das Abkommen. Weniger klar ist jedoch, inwiefern ein Patentinhaber die nicht bewilligte Einfuhr patentierter Produkte, die er auf ausländischen Märkten verkauft, verhindern kann.

๖ Zwangslizenzen dürfen nur unter strengen Bedingungen vergeben werden. Die automatische Vergabe von Zwangslizenzen - z.B. für alle pharmazeutischen Erfindungen - ist nicht mehr möglich.

\ Die Durchsetzung von Patentrechten erfolgt unabhängig vom Technologiebereich. Das beinhaltet sowohl das Verbot von Zwangslizenzen für einen bestimmten Sektor wie z.B. Arzneimittel als auch von anderen Formen der Diskriminierung. Gesetze, welche während der Patentdauer Tätigkeiten erlauben, die ansonsten eine Rechtsverletzung darstellen und die ermöglichen, dass unmittelbar nach Erlöschen des Patentschutzes ein Generikum des Medikaments hergestellt wird, laufen dem TRIPs-Abkommen zuwider.

๑ Gegen jede Entscheidung zum Widerruf eines Patents muss ein Rechtsmittel eingelegt werden können.

๑ Die Schutzdauer beträgt ab dem Tag der Einreichung mindestens 20 Jahre. Gemäss den Übergangsbestimmungen des TRIPs gilt dies auch für zum Zeitpunkt der Gesetzesänderung anhängige Patentanmeldungen sowie für bereits erteilte Patente.

๑ Für Verfahrenspatente ist eine Umkehrung der Beweislast erforderlich. Wird z.B. ein neues Produkt durch ein Patent geschützt, dass sich nur auf das Herstellungsverfahren bezieht, so gilt ein identisches nachgeahmtes Erzeugnis bis zum Beweis des Gegenteils durch den Nachahmer als durch das patentierte Verfahren hergestellt. 


\subsection{SCHUTZ VERTRAULICHER INFORMATIONEN}

Die Bestimmung, wonach Mitgliedsstaaten aufgefordert werden, die vertraulichen Registrierungsangaben vor unlauterer gewerblicher Verwendung zu schützen, betrifft zwar nicht direkt Patente, ist aber für die pharmazeutische Industrie von besonderem Interesse. Zahlreiche Industrieländer, darunter die Schweiz, gewähren noch keinen ausschliesslichen Schutz für solche Angaben. Andere verknüpfen die Schutzdauer mit der Patentdauer, was den Wert des Schutzes erheblich verringert und als TRIPs-widrig gelten sollte, zumal die fragliche Bestimmung keine Beziehung zum Abschnitt über die Patente besitzt. Das TRIPs-Abkommen schreibt keinerlei Dauer des Informationsschutzes vor. Die pharmazeutische Industrie schlägt eine weltweite Standardschutzdauer von 10 Jahren ab der Registrierung in jedem Land vor.

\subsection{BESTIMMUNGEN ZUR DURCHSETZUNG DER RECHTE AM GEISTIGEN EIGENTUM}

Mit der Durchsetzung der geistigen Eigentumsrechte, einschliesslich der Patente, befasst sich ein ganzer Abschnitt des TRIPs-Abkommens. Die Mitglieder müssen effiziente, gerechte und nicht unnötig kostspielige Durchsetzungsverfahren vorsehen. Ferner müssen ordentliche Verfahren gewährleistet sein: Anspruch auf einen Rechtsbeistand, Recht, sich zu allen entscheidungsrelevanten Beweismitteln zu äussern, Recht, Entscheidungen der Verwaltung durch die Justizbehörde überprüfen zu lassen.

In Prozessen zu Fragen des geistigen Eigentums müssen die Justizbehörden befugt sein, das Beibringen von Beweismitteln zu verlangen, Verfügungen zu erlassen, Kosten und Entschädigungen festzusetzen oder die Zerstörung oder Beschlagnahme rechtsverletzender Waren anzuordnen. In bestimmten Fällen muss der Richter ohne Benachrichtigung der beklagten Partei einstweilige oder vorläufige Verfügungen erlassen können. Die Mitglieder haben die erforderlichen Vorkehrungen zu treffen, damit die Zollbehörden die Einfuhren gefälschter Markenwaren oder unerlaubt hergestellter urheberrechtlich (aber nicht unbedingt patentrechtlich) geschützter Waren auf Verlangen verhindern können. Zumindest für die vorsätzliche und gewerbsmässige Fälschung von Markenwaren und urheberrechtlich geschützten Waren sollen strafrechtliche Sanktionen eingeführt werden; für andere Arten von Verstössen gegen geistige Eigentumsrechte sind Strafen fakultativ.

\subsection{ZUSAMMENFASSUNG}

Dank dem TRIPs-Abkommen sind fast alle Länder der Welt verpflichtet, Patentsysteme vorzusehen, die pharmazeutische Verbindungen während mindestens 20 Jahren schützen - ohne Auflagen zur lokalen Herstellung und ohne routinemässige Vergabe von Zwangslizenzen. Die Einfuhr eines Erzeugnisses und der Verkauf eines auf patentierte Verfahren zurückgehenden Erzeugnisses werden eindeutig als Verstoss definiert. Für die Durchsetzung von Patentrechten gelten klare Standards. Das bedeutet nicht nur einen grossen Fortschritt für die forschungsgestützte Pharmaindustrie, sondern auch eine echte Chance für Entwicklungsländer, geistige Eigentumsrechte einzuführen, die ihre wirtschaftliche Entwicklung fördern. 
Eine wichtige Rolle spielt auch das neue Streitbeilegungssystem des GATT, das Sanktionen gegen Mitgliedsstaaten ermöglicht, die ihre TRIPs-Verpflichtungen verletzt haben. Die Industrie bedauert, dass die Streitbeilegungsverfahren nicht auch auf Fälle anwendbar sind, die sich auf die Erschöpfung von Patentrechten beziehen: Laut dieser Theorie gelten Patentrechte durch den Verkauf eines patentrechtlich geschützten Produkts direkt durch den Patentinhaber oder mit seiner Zustimmung in jedem Land (selbst dort, wo es kein Patent gibt) als «erschöpft», und die unerlaubte Einfuhr dieser Produkte in ein anderes Land kann selbst durch das Bestehen eines Patentes in diesem Land nicht verhindert werden. Ein freier Warenverkehr dieser Art sollte nur in einem Binnenmarkt gestattet werden, wo es anders als in der EU keine durch staatliche Preispolitik verursachten Marktverzerrungen gibt.

\section{2. ÄNDERUNGEN DER PATENTRECHTE ALS FOLGE DES TRIPS}

Am 1. Januar 1995 gab es in fast allen WTO-Mitgliedsstaaten noch Gesetze zum geistigen Eigentum, die in verschiedener Form gegen das TRIPs-Abkommen verstiessen. In zahlreichen Staaten - Industrie- und Entwicklungsländern lag die Patentdauer unter den im TRIPs geforderten 20 Jahren ab dem Hinterlegungsdatum (oder konnte zumindest darunter liegen). Dieser Mangel liess sich verhältnismässig leicht beheben, obwohl einige Länder dies erst spät taten. Im Fall von Ländern wie Indien, Argentinien, Brasilien und der Türkei fehlte ein Patentschutz für Arzneimittel (fast) ganz, so dass umfassende Revisionen notwendig waren; in der Zwischenzeit sollten die sogenannten «Black BoxBestimmungen» gelten (siehe Abschnitt 3 unten). Selbst Industrieländer wie die Vereinigten Staaten, EU-Länder und Japan kannten Gesetze, die auf subtilere Weise - etwa mit Bedingungen für die Erteilung von Zwangslizenzen - dem TRIPs zuwiderliefen. Alle diese Gesetze müssen, sofern sie noch nicht geändert worden sind, in Übereinstimmung mit dem TRIPs gebracht werden. Dafür gilt folgender Zeitplan :

\section{1. ÜBERGANGSBESTIMMUNGEN DES TRIPS}

\section{$\square$ Umsetzungsfristen}

Kein Land ist verpflichtet, seine Gesetze vor Ablauf der Frist von einem Jahr nach der Errichtung der WTO - also am 1. Januar 1996 - zu ändern und mit dem TRIPs in Einklang zu bringen. Zwei Hauptgruppen von Ländern haben die Möglichkeit, für die Anpassungen einen längeren Zeitraum in Anspruch zu nehmen. Gemäss den Übergangsvereinbarungen sind die Entwicklungsländer berechtigt, die Anwendung des TRIPs um eine weitere Frist von vier Jahren, also bis zum 1. Januar 2000, zu verschieben. Gleiches gilt für Länder, die sich im Übergang von der Planwirtschaft zur Marktwirtschaft befinden und die bei der Einführung des Systems der geistigen Eigentumsrechte Problemen gegenüberstehen. Ungarn, Polen und die Tschechische Republik zum Beispiel, die bereits angemessene Patentsysteme besitzen, gehören nicht dazu.

Entwicklungsländer, die am 1. Januar 2000 bestimmte Technologiesektoren (z.B. Arzneimittel) noch nicht schützen, können die Einführung des Stoffschutzes in diesen Bereichen um weitere fünf Jahre, also bis zum 1. Januar 
2005, aufschieben (allerdings dürfen sie sich nicht den übrigen Verpflichtungen aus dem TRIPs entziehen). Die am wenigsten entwickelten WTO-Mitglieder schliesslich sind berechtigt, die Anwendung aller TRIPs-Bestimmungen mit Ausnahme der in Absatz 1.2 oben angeführten Grundbestimmungen bis zum 1. Januar 2006 auszusetzen, wobei selbst diese Frist auf besonderen Antrag noch verlängert werden kann.

2006 liegt zwar noch weit weg. Die klar definierte Gruppe der laut offizieller UN-Liste 49 Länder dürfte aber angesichts ihres heutigen Entwicklungsstandes auch in zehn Jahren noch keine bedeutenden Märkte darstellen. Pharmazeutische Unternehmen können nicht in jedem Land der Welt Patente hinterlegen, und eine Verbesserung des Patentschutzes in den am wenigsten entwickelten Länder dürfte von geringer praktischer Bedeutung sein. Was allerdings die «Entwicklungsländer» anbelangt, so gibt es unseres Wissens keine objektiven Definitionskriterien. Verschiedene wichtige Wirtschaftspartner haben sich im Zusammenhang mit dem TRIPs selbst als «Entwicklungsländer» definiert, obwohl sie sich normalerweise nicht als solche betrachten.

\subsection{ANPASSUNG DER PATENTDAUER}

Die Angleichung der Patentdauer als Anpassung an die TRIPs-Bestimmungen schafft keine nennenswerten Schwierigkeiten. Die Mehrheit der WTO-Mitglieder, so Argentinien, Brasilien, Indonesien und die Philippinen, haben dies bereits getan. Malaysia hat die Frist von 15 Jahren ab der Patenterteilung noch nicht verlängert. Korea hingegen hat das betreffende Gesetz geändert: vorher betrug die Schutzdauer 20 Jahre ab der Hinterlegung oder 15 Jahre ab der Offenlegung für Einspruch, je nach kürzerer Frist. Die zweite Alternative wurde aufgehoben, aber die Änderung gilt erst seit dem 1. Juli 1996. Das neue koreanische Patentrecht verlängert die Dauer der zu diesem Zeitpunkt gültigen Patente; Patente jedoch, die im ersten Halbjahr 1996 ausgelaufen sind und hätten verlängert werden müssen, erlöschen definitiv. In anderen Ländern, z.B. Argentinien und Brasilien, wurde die Dauer der gültigen Patente nicht verlängert. Damit befassen sich heute die Gerichte.

\subsection{ZWANGSLIZENZEN}

Das TRIPs-Abkommen schreibt für Zwangslizenzen folgendes vor:

Sie müssen nach den Umständen des Einzelfalls geprüft werden;

Sie dürfen nur dann erteilt werden, wenn eine Lizenz zu normalen Bedingungen beantragt und verweigert wurde;

Umfang und Dauer sind begrenzt;

Sie sind nicht übertragbar, ausser im Zusammenhang mit dem ganzen Geschäft ; Sie sind vorwiegend für den Binnenmarkt, nicht für den Export, bestimmt;

Ihre Aufhebung ist möglich, wenn die Umstände sich ändern;

Bezahlung einer angemessenen Vergütung;

Sie unterliegen der Überprüfung durch ein Gericht.

Zwangslizenzen zur Verwendung eines abhängigen Patents dürfen nur erteilt werden, wenn die darin beanspruchte Erfindung einen erheblichen technischen Fortschritt von wesentlicher wirtschaftlicher Bedeutung beinhaltet, und wenn 
die Zwangslizenz, sofern sie erteilt wird, ausser bei Übertragung des abhängigen Patents nicht übertragbar ist. Ende 1995 zeigte die Untersuchung der GD XV der Europäischen Kommission, dass in keinem einzigen EU-Mitgliedsstaat das Recht diese Bestimmungen lückenlos erfüllte. Insofern überrascht es nicht, wenn die Entwicklungsländer in dieser Hinsicht oft noch viel zu wünschen übrig lassen. Allerdings steht fest, dass die diskriminierende Erteilung von Zwangslizenzen auf pharmazeutischen Patenten beendet werden muss. Problematisch erscheint auch, dass manche TRIPs-Bestimmungen zu Zwangslizenzen nicht auf jene Zwangslizenzen anwendbar sind, die zur Bekämpfung von wettbewerbswidrigen Praktiken erteilt werden. Durch dieses Schlupfloch könnten die Bestimmungen umgangen werden, indem Verwaltungsbehörden das Verhalten des Patentinhabers ganz einfach als "wettbewerbswidrig» erklären. Im schlimmsten Fall kann der Nachahmer ein patentiertes Produkt billiger anbieten: der Patentinhaber, der die Verletzung beanstandet, wird als wettbewerbswidrig eingestuft und der Nachahmer erhält eine Zwangslizenz.

\subsection{EINFÜHRUNG DES STOFFSCHUTZES FÜR ARZNEIMITTEL}

Dieser Punkt bereitet für die Anwendung des TRIPs-Abkommens besondere Schwierigkeiten. Wie oben erwähnt, können die Entwicklungsländer zwar dafür eine Frist bis zum Jahr 2005 in Anspruch nehmen, müssen aber zwischenzeitlich «Black Box»-Bestimmungen umsetzen (siehe unten). Die pharmazeutische Industrie stellt sich aber die berechtigte Frage, ob Länder wie Argentinien oder die Türkei, die noch keinen Stoffschutz für Arzneimittel eingeführt haben, zu Recht als Entwicklungsländer gelten sollen - «in Entwicklung» befinden sich schliesslich alle Länder, sogar die Schweiz.

\section{BLACK BOX-ANMELDUNGEN}

Bei den sogenannten «Black Box»-Anmeldungen handelt es sich um ein wichtiges Konzept der Übergangsbestimmungen des TRIPs. Die Bestimmungen werden in manchen Ländern immer noch ignoriert oder falsch angewandt.

Wenn ein Mitgliedsstaat nicht ab dem Inkrafttreten des WTO-Abkommens einen Patentschutz für pharmazeutische und agrochemische Produkte vorsieht, so muss er ab diesem Zeitpunkt (d.h. ab dem 1. Januar 1995 oder später zum Zeitpunkt seines Beitritts) ein System anbieten, das die Einreichung von Anmeldungen für solche Erfindungen erlaubt.

Für solche Anmeldungen gelten ab der Anwendung des Abkommens (d.h. wie oben erläutert ab Beginn der Geltung der Verpflichtungen aus Teil II Abschnitt 5 TRIPs) die TRIPs-Kriterien zur Patentfähigkeit, und zwar so, als ob sie beim Zeitpunkt der Anmeldung in diesem Land gelten würden.

Das System muss ab der Erteilung einen Patentschutz für die restliche Patentdauer (d.h. 20 Jahre ab dem Hinterlegungsdatum) gewähren, wenn die Erfindung gemäss den TRIPs-Kriterien patentfähig ist. Zudem muss ein Produkt, für welches eine «Black Box»-Anmeldung gewährt wurde, ausschliessliche Vermarktungsrechte für fünf Jahre ab der Marktzulassung im fraglichen Land oder bis zur Erteilung oder Verweigerung eines Stoffschutzpatentes (je nach kürzerer 
Frist) erhalten. Vorausgesetzt wird, dass für das Produkt in einem anderen Mitgliedsstaat a) eine Patentanmeldung nach dem 1.1.1995 hinterlegt wurde, b) ein Patent erteilt und c) die Marktzulassung gewährt wurde. Es handelt sich um komplexe Bestimmungen, die eine Zusammenarbeit der nationalen Patentämter und der Gesundheitskontrollbehörden erfordern.

Die komplizierten «Black Box»-Bestimmungen spielen eine sehr wichtige Rolle, da sie bis zum Inkrafttreten neuer Gesetze die einzige Möglichkeit bilden, auf wichtigen Märkten wie Indien, Pakistan, der Türkei und Argentinien einen Patentschutz für pharmazeutische Produkte zu erzielen.

Die Analyse lässt ersehen, dass - selbst wenn es nicht explizit formuliert wird Anmeldungen für pharmazeutische Verbindungen nicht geprüft werden (ausgenommen vielleicht eine förmliche Untersuchung zum Nachweis, dass die Anmeldung ein Einreichungsdatum erhalten kann), bis das fragliche Land sein Recht den TRIPs-Bestimmungen zur Patentfähigkeit von pharmazeutischen Produkten angeglichen hat. Gerade deswegen werden solche Anmeldungen ja als «Black Box-Filings» bezeichnet. Überdies ist klar, dass alle Mitglieder ab dem 1. Januar 1995 bzw. ab ihrem späteren Beitritt zur WTO diese Einreichungsmöglichkeit vorsehen müssen. Manche Länder allerdings stecken die Anmeldungen entweder gar nicht in die «Black Box» oder holen sie viel zu früh wieder heraus.

Ein Beispiel ist Indien. In Indien herrscht eine Auseinandersetzung zwischen jenen Politikern (der früheren und der aktuellen Regierung), welche eine Öffnung der indischen Volkswirtschaft nach aussen befürworten und einen WTOBeitritt als wichtige Etappe auf diesem Weg sehen, und anderen Kräften, die mit starkem populärem Rückhalt am alten Protektionismus festhalten und sich gegen jegliche Änderung des völlig unzureichenden Patentrechts sperren. So hat Indien die GATT-Übereinkommen am 31. Dezember 1994 durch ein Regierungsdekret unterzeichnet, ohne die Zustimmung des indischen Parlaments einzuholen, und hat gleichzeitig mit einer Änderung des Patentrechts (Patent Amendment Ordinance) «Black Box-Filings » ermöglicht.

Allerdings trat diese Verordnung später ausser Kraft, und ein neues Gesetz scheiterte an der Zustimmung des Parlaments. Als Ergebnis werden «Black Box »-Anmeldungen zwar weiterhin toleriert, aber nicht mehr offiziell anerkannt. Zudem ist ihr rechtlicher Status unklar. Man nimmt fälschlicherweise immer noch an, für «Black Box»-Anmeldungen seien besondere Ausführungsgesetze erforderlich. Das ist eindeutig nicht der Fall, unabhängig davon, ob die indische Verfassung ein selbständiges Inkrafttreten des GATT-Vertrags vorsieht oder nicht. Die Einreichungen können im Rahmen der geltenden Gesetze als normale Einreichungen behandelt werden und anschliessend durch eine rein administrative Massnahme so lange anhängig bleiben, bis eine Gesetzesänderung beschlossen wird - falls es je soweit kommt. Die Bestimmungen zu den ausschliesslichen Vermarktungsrechten (EMR) in den Übergangsvereinbarungen des TRIPs wurden besonders entschieden abgelehnt. Obwohl die Verordnung selbst im Widerspruch zum TRIPs Zwangslizenzen und die Preisfestsetzung für ausschliessliche Vermarktungsrechte vorsieht, wurde in mehreren Vorschlägen eine weitere Schwächung der Auswirkungen gefordert. Heute dreht sich die Diskussion hauptsächlich um die Anwendung der Übergangsbestimmungen in Indien. Hinsichtlich einer umfassenden Überarbeitung des indischen 
Patentrechts, um es in Einklang mit den TRIPs-Grundsätzen zu bringen, werden geringe oder keine Fortschritte verzeichnet. Derzeit ist in der WTO ein von den USA beantragtes und von Indien akzeptiertes Streitbeilegungsverfahren im Gange. Das WTO-Streitbeilegungsorgan hat kürzlich eine Entscheidung gegen Indien erlassen, gegen die Indien Berufung einlegen kann.

Indien besitzt sicher das schlechteste Patentrecht wenn nicht weltweit, so doch unter den wirtschaftlich wichtigen Ländern. Für pharmazeutische Erfindungen kann nur ein Herstellungsverfahren eines Produkts patentiert werden, obwohl die zahlreichen kompetenten indischen Chemiker leicht neue Verfahren erfinden könnten. Die Dauer ist auf 7 Jahre begrenzt: Das Patent würde also vor der möglichen Einführung des Produkts in Indien erlöschen, und sollten dennoch einige Patente übrigbleiben, so werden Zwangslizenzen für eine Gebühr von $4 \%$ des Grosshandelspreises erteilt - wobei die «Gebühr» eine verschleierte Beschlagnahme darstellt.

Indische Aktivisten beschweren sich, wenn westliche Unternehmen eine neue Zusammensetzung des indischen Nimbabaums patentieren (ausser natürlich wenn dies in Indien geschieht). Statt dessen sollten sie sich fragen, weshalb kein indisches Unternehmen in Forschung für neue Derivate der traditionellen indischen Medizin investiert. Die Anwort liegt auf der Hand: In Indien könnten sie ihre Erfindungen nicht patentieren lassen, und andere Unternehmen könnten sie ungehindert kopieren. Indien sollte seine postkoloniale Mentalität endlich ablegen und sich den anderen Ländern Asiens anschliessen, die erkannt haben, dass Wirtschaftsentwicklung und starker Patentschutz Hand in Hand gehen.

Auch Pakistan hat bis vor sehr kurzem «Black Box»-Anmeldungen unter dem Vorwand fehlender Ausführungsgesetze abgelehnt. Vor kurzem hat jedoch das Parlament einen Gesetzesentwurf zur Einführung der «Black Box-Filings » gutgeheissen, der rückwirkend für ab dem 1. Januar 1995 hinterlegte Einreichungen gelten soll.

Die Türkei umgeht TRIPs-Bestimmungen zur «Black Box» auf eine andere Weise. Die Türkei akzeptiert zwar Einreichungen für pharmazeutische Produkte; trotz der unveränderten Rechtslage werden die Anmeldungen aber nicht einer «Black Box» zugewiesen, sondern denselben überaus kostspieligen Recherchen wie Anmeldungen in anderen Technologiesektoren unterworfen. Positiv ist dabei wenigstens, dass es im Moment der Gesetzesänderung nur noch wenige nicht untersuchte Anmeldungen geben wird. Zuerst hatte die Türkei vorgeschlagen, die Erteilung von Verfahrenspatenten für Arzneimittel um 5 Jahre und von Stoffschutzpatenten um 10 Jahre aufzuschieben. Dieses Vorhaben liess sich schwer mit dem türkischen Gesuch um Beitritt zur Zollunion und langfristig zur Vollmitgliedschaft in der Europäischen Union vereinbaren. Heute geht man davon aus, dass die Türkei spätestens im Jahr 1999 der Europäischen Patentkonvention beitreten wird.

Brasilien ist eine «Erfolgsstory» in der Geschichte des GATT/TRIPs. «Black Box-Filings » wurden von Anfang an problemlos akzeptiert. Am 15. März 1997 ist das neue Patentrecht in Kraft getreten, das einen Stoffschutz für Arzneimittel vorsieht. Mit Ausnahme der weiterhin geltenden lokalen Herstellungsvorschriften stimmt das neue Patentrecht voll mit dem TRIPs überein. In zwei Punkten geht es sogar über die TRIPs-Anforderungen hinaus: Brasilien sieht ein Pipeline-Schutzsystem (vorübergehender Schutz) vor und erlaubt das Verhindern 
von Parallelimporten. Negativ zu bewerten ist, dass die 20jährige Schutzdauer des TRIPs noch nicht auf bereits erteilte Patente ausgedehnt wurde.

Argentinien ist leider ein ganz anderer Fall. Zwar befürwortete die Regierung allgemein eine Verbesserung des Patentschutzes, aber das Parlament war massivem Lobbying der lokalen «Pharmaindustrie» ausgesetzt - sprich einer kleinen Gruppe profitorientierter Unternehmen, die mit dem Verkauf von Generika oder Kopien von aus Ländern wie Indien importierten patentierten Arzneien Geld machen. Solche Unternehmen tragen in der Tat wenig zur Wertschöpfung innerhalb des Landes bei.

Als Ergebnis wurde nach einer endlosen Abfolge neuer Gesetze, präsidialer Vetos, «Korrektur-Gesetzesentwürfen» usw. ein neues Gesetz verabschiedet, das im Januar 1997 in Kraft getreten ist. Darin wird der Stoffschutz bis zum 1. Januar 2001 hinausgeschoben (Argentinien soll also als Entwicklungsland betrachtet werden). Darüber hinaus läuft das Gesetz in verschiedenen weiteren Punkten dem TRIPs zuwider. «Black Box»-Anmeldungen sind zwar möglich, aber es fehlt eine Bestimmung zur ausschliesslichen Vermarktung, die gerade dann besonders wichtig ist, wenn wie in Argentinien der Stoffschutz erst sehr spät umgesetzt wird.

\section{ERTEILUNG UND DURCHSETZUNG VON PATENTEN}

Durchschlagende Patentgesetze auf dem Papier bleiben wirkungslos, wenn Anmeldungen nicht auch bearbeitet, Patente erteilt und durchgesetzt werden können. Zahlreiche Entwicklungsländer haben kürzlich ihr Patentrecht verbessert. Die Gesetze setzen oft eingehende Untersuchungen bezüglich der Neuheit und der erfinderischen Tätigkeit des angemeldeten Produkts oder Verfahrens voraus. Die Länder befinden sich in einer schwierigen Lage: Wegen der verbesserten Gesetze erhalten die Patentämter mehr Anmeldungen als zuvor, können aber aus Mangel an technischem Personal und an Infrastrukturen die Anmeldungen nicht in der gesetzlich verlangten Weise prüfen. Das Problem darf nicht unterschätzt werden. Um eine vertiefte Untersuchung in allen technischen Bereichen durchzuführen, muss ein Patentamt über zahlreiche qualifizierte technische Mitarbeiter und erhebliche Ressourcen an wissenschaftlicher und patentrechtlicher Literatur, Zugang zu elektronischen Datenbanken usw. verfügen. Dazu dürften weltweit nur ungefähr 10 Patentämter in der Lage sein. Die Aufgabe übersteigt die Mittel der meisten Entwicklungsländer bei weitem; zudem würden sie sinnlos Ressourcen verschwenden, wenn sie Arbeiten doppelt leisten müssten, die in den meisten Fällen schon von amerikanischen, europäischen oder japanischen Patentämtern durchgeführt wurden.

Organisationen wie das EPA und die Weltorganisation für geistiges Eigentum (WIPO oder OMPI in Genf) unterstützen die Patentämter in Entwicklungsländern bei der Bewältigung der Anmeldungsflut. Unabhängige technische Prüfungen sollen dabei nach Möglichkeit vermieden werden. Patente werden aufgrund der Annahme entsprechender Patente in einem wichtigen Patentamt oder, wenn das fragliche Land dem Vertrag über die internationale Zusammenarbeit auf dem Gebiet des Patentwesens (PCT) beigetreten ist, aufgrund des internationalen vorläufigen Prüfungsberichts des PCT erteilt. 
Zur Durchsetzung der Patente bedarf es kompetenter Gerichte und Richter, die sich im Patentrecht auskennen. Noch wichtiger ist wohl ein rechtliches Umfeld, in welchem Verletzungen der geistigen Eigentumsrechte als Missstand beurteilt und mit hohen Geldbussen bestraft werden, und nicht als lokale Wachstumsindustrie gelten, die man ignorieren oder gar fördern darf.

Mexiko zum Beispiel verfügt seit 1992 über ein ausgezeichnetes, weitgehendes Patentrecht. Da jedoch Ausführungsvorschriften fehlten, sind bis vor kurzem keine Patente erteilt worden. Diese Lücke wird heute behoben, so dass die Erteilung von Patenten möglich ist. Die Durchsetzung solcher Patente gegenüber lokalen Nachahmern erweist sich allerdings als äusserst schwierig.

Dasselbe gravierende Problem existiert auch in China, das zwar ein durchaus angemessenes Patentrecht besitzt, aber die Tradition der gerichtlichen Beilegung von Streitigkeiten nicht kennt. Patentfälle können theoretisch vor das Volksgericht (People's Court) gebracht werden. Meistens jedoch werden sie in einem administrativen Verfahren vor der Verwaltungsbehörde für Patentangelegenheiten behandelt. Nicht selten stellt sich dabei heraus, dass das rechtsverletzende Unternehmen Eigentum der Verwaltungsbehörde oder einer anderen staatlichen Stelle ist. Effektive Massnahmen zur Unterbindung der Rechtsverletzung bleiben so höchst unwahrscheinlich.

\section{MÖGLICHE ÄNDERUNGEN DES TRIPS-ABKOMMENS}

Laut Artikel 27 Absatz 3 (b) können die Mitgliedsstaaten Pflanzensorten und Tierarten von der Patentfähigkeit ausschliessen, vorausgesetzt, dass die Pflanzensorten durch Patente oder durch ein besonderes Schutzsystem wie die Sortenschutzrechte (Plant Breeders'Rights) geschützt sind. Dieser Unterabsatz wird vier Jahre nach Inkrafttreten der Abkommens zur Errichtung der WTO, also am 1. Januar 1999, überprüft. Unklar ist, ob die Überprüfung zu diesem Zeitpunkt begonnen oder abgeschlossen werden soll. Die erste Variante gilt jedoch als wahrscheinlicher.

Aus der Perspektive der Industrie muss sich die Überarbeitung einzig auf die Frage beschränken, ob es erlaubt bleibt, Pflanzen und Tiere von der Patentfähigkeit auszuschliessen. Es ist zu hoffen, dass die Mitglieder nach der vierjährigen «Gnadenfrist» aufgefordert werden, einen Patentschutz für mehrzellige lebende Organismen vorzusehen (Mikroorganismen sollten in jedem Fall patentfähig sein). Allerdings möchten zahlreiche NGOs und Umweltorganisationen sowie Entwicklungsländer wie Indien von diesem Ansatzpunkt aus ganze Bereiche des TRIPs-Abkommens neu verhandeln. So wird insbesondere auf den vorhergehenden Absatz verwiesen, Art. 27 Abs. 2, wonach die Mitglieder «Erfindungen von der Patentfähigkeit ausschliessen können, wenn die Verhinderung ihrer gewerblichen Verwertung in ihrem Hoheitsgebiet zum Schutz der öffentlichen Ordnung oder der guten Sitten einschliesslich des Schutzes des Lebens oder der Gesundheit von Menschen, Tieren oder Pflanzen oder zur Vermeidung einer schweren Schädigung der Umwelt notwendig ist». Ferner sind Bestrebungen im Gange, das TRIPs-Abkommen mit dem Übereinkommen über die biologische Vielfalt zu verknüpfen. Anti-Patent-Kreise hoffen, so das TRIPs-Abkommen abzuändern, um beispielsweise die Patentfähigkeit menschlicher Gene (die als 
«unmoralisch» gilt), die Untersuchung von Pflanzen und Mikroorganismen auf verwertbare Verbindungen («Bio-Piraterie»), kurz, jeden möglicherweise umweltschädlichen Akt, zu verhindern.

Erstens ist anzumerken, dass weder Artikel 27 Absatz 2 noch ein anderer Teil des TRIPs, ausgenommen Artikel 27 Absatz 3 (b), der obligatorischen Überprüfung untersteht.

Zweitens befasst sich das Übereinkommen über die biologische Vielfalt mit völlig unterschiedlichen Gesetzen als das TRIPs-Abkommen. Das Übereinkommen über die biologische Vielfalt geht von den souveränen Vorrechten der Staaten über ihre eigenen biologischen Ressourcen aus. Ziele sind die Erhaltung der biologischen Vielfalt, die nachhaltige Nutzung ihrer Bestandteile und - dank Technologietransfer und Zugang zu Ressourcen - die gerechte Aufteilung der Vorteile (Art. 1 Abs. 1). Das TRIPs-Abkommen hingegen verpflichtet die Staaten, das als privates Recht anerkannte geistige Eigentum zu schützen. Beide Systeme sind vollkommen miteinander vereinbar, und sie dürfen nicht angewandt werden, um ihre Ziele gegenseitig zu unterlaufen. So fordert das Übereinkommen über die biologische Vielfalt in bezug auf den Technologietransfer, dass der Zugang und die Weitergabe von Technologie zu Bedingungen erfolgen, die einen angemessenen und wirkungsvollen Schutz der Rechte am geistigen Eigentum anerkennen und mit ihm vereinbar sind (Art. 16 Abs. 2). Die Vertragsparteien haben sicherzustellen, dass vorbehaltlich des innerstaatlichen Rechts und des Völkerrechts (z.B. TRIPs-Abkommen) geistige Eigentumsrechte die Ziele des Übereinkommens unterstützen und ihnen nicht zuwiderlaufen (Art. 16 Abs. 5). Die Industrie ist überzeugt, dass ein wirksamer Patentschutz den Technologietransfer fördert.

Drittens bedeutet die mögliche Ausschliessung von der Patentfähigkeit aus Gründen der «ordre public» (öffentliche Ordnung) oder der guten Sitten keineswegs, dass jede Interessengruppe Druck ausüben und die Patenterteilung für Erfindungen blockieren kann, nur weil sie diese ablehnen. Die Möglichkeit der Ausschliessung von der Patentfähigkeit besteht nur dann, wenn «die Verhinderung» der gewerblichen Verwertung der Erfindung «notwendig ist». Die Patentfähigkeit ist allerdings selbst dann nicht automatisch ausgeschlossen, wenn das inländische Gesetz die Nutzung der Erfindungen verbietet. Daraus folgt, dass bei legaler kommerzieller Nutzung Patente erteilt werden müssen. 